\title{
A New RF Power Amplifier Linearization Method
}

\author{
Zhong-Yang Mao \\ China Electronic System Corp, Beijing 100141, China \\ E-mail: freedom_mzy@163.com
}

\author{
Xiao-jun Wu \\ Unit 92514 of the PLA, Shantou Guangdong 515074, \\ China \\ E-mail: 195358836@qq.com
}

\author{
Fa-Ping Lu, Min Fan, Zhong-Yang Mao, Chuan-Hui Liu \\ Department of Electrical and Information Engineering, Naval Aeronautical and Astronautical University, Yantai 264001, \\ China \\ Key Laboratory on Signal \& Information Processing of Shandong Provience, Yantai 264001, China \\ E-mail: lufaping@163.com
}

\begin{abstract}
For the weakness of the existing predistortion algorithm, when large amplitude component in the modulation signal which is more than the voltage in saturation will be limited, leading to predistortion algorithm become invalid, and a new joint RF power amplifier (PA) linearization method is proposed. The method in this paper combines Peak-to-average Power Ratio (PAPR) reduction method base on $u$-law with predistortion algorithm, first compressing the signal amplitude by using PAPR reduction method, then linearizing the modulation signal by using predistortion algorithm, two boxes supplemented to each other, solving the weakness of the existing predistortion algorithm. The simulation result shows that the proposed method can effectively improve the power spectrum of the signal with about $5 \mathrm{~dB}$ out-of-band distortion compensation, effectively improve the Bit-Error-Ratio (BER) performance of system, and reduce the gap to the original signal less than $2 \mathrm{~dB}$ when the BER performance is $10^{-5}$ in nonlinear AWGN channel.
\end{abstract}

Keywords-power amplifier; signal compression; predistortion algorithm; joint

\section{INTRODUCTION}

Prolate Spheroidal Wave Function is Bell Laboratories D. Slepian and O. Pollak defined a collection class of special functions in $1961^{[1]}$, which has been widely used in Communications Engineering ${ }^{[2]}$, fluid dynamics ${ }^{[3]}$, and other fields. Based on the existing properties of PSWF, such as the biorthogonal features, spectrum controllability, the best time-frequency energy clustering, etc. The literature [4] proposes a non-sinusoidal time-domain orthogonal modulation method based on PSWF, which improves the bandwidth efficiency by waveform superimposition in time domain and frequency spectrum overlapping in frequency domain, making the bandwidth efficiency can quickly approaching $2 \mathrm{baud} / \mathrm{Hz}$ theory of Shannon limit. But as a result of using multichannel parallel, the problem of high PAPR exists in the modulation signal, which leading signal affected by PA vulnerably, reducing the performance of the system. Therefore, the efficient predistortion method becomes the key to guarantee the performance of the commination system based on PSWF.
At present, there is less research about the predistortion algorithm for PSWF modulation signal at home and abroad relative to OFDM modulation signal. Zhong et.al. researches the characteristics of PSWF modulation signal and digital predistortion technology deeply, and on this basis, two predistortion algorithms for PSWF modulation signal are proposed, one is predistortion algorithm based on compressing quantization ${ }^{[5]}$, and the other is predistortion algorithm based on parallel two-box model ${ }^{[6]}$. From the perspective of reducing the highest order of polynomial, Lu et.al. combine with the recursive least squares algorithm, presents a predistortion method for PSWF modulation signal based on least squares algorithm ${ }^{[7]}$. But for the problem of the existing predistortion algorithm for PSWF modulation signal, when large amplitude component in the modulation signal which is more than the voltage in saturation will be limited, leading to predistortion algorithm become invalid, there are no methods proposed for this weakness of the existing predistortion algorithm for PSWF modulation signal.

From the perspective of compressing the large amplitude component in PSWF modulation signal, combining with PAPR reduction method base on $u$-law ${ }^{[8]}$, a new joint RF PA linearization method is proposed. The method in this paper consists of two boxes, one is PAPR reduction model, the other is predistortion algorithm model, two boxes supplement to each other. The simulation result shows that the proposed method can effectively solving the weakness of the existing predistortion algorithm.

\section{JOINT RF POWER AMPLIFIER LINEARIZATION METHOD}

\section{A. The Model of Joint RF Power Amplifier Linearization Method}

At present, PA model mainly divided into memoryless PA model and memory PA model, not considering the memory effect of PA, choosing the Saleh model which is widely used in the satellite communication system. Saleh model is based on the statistical analysis of input and output data from the traveling wave tube power amplifier TWTA. 
The AM-AM and AM-PM features of Saleh model can be expressed as respectively[9]

$$
A(r)=\frac{\alpha_{a} r}{1+\beta_{a}^{2} r^{2}}, \quad \Phi(r)=\frac{\alpha_{\psi} r^{2}}{1+\beta_{\psi} r^{2}}
$$

Where, $\mathrm{r}$ is amplitude of the input signal, $\alpha \mathrm{a}, \beta \mathrm{a} 2, \alpha \psi$, $\beta \psi$ are the parameters of PA model. According to formula (1), we know that

$$
A(r)=\frac{\alpha_{a}}{1 / r+\beta_{a}^{2} r} \leq \frac{\alpha_{a}}{2 \beta_{a}}
$$

Where, $1 / \mathrm{r}=\beta \mathrm{a} 2 \mathrm{r}$ is the condition for equality. According to formula (1), we know that the large amplitude component in the modulation signal which is more than the voltage $\beta \mathrm{a}$, its amplitude will be limited when signal is passed through PA, leading to predistortion algorithm become invalid, reducing BER performance of the system.

According to formula (1) and (2), we know that if we want to avoid large amplitude component being limited, the large amplitude component in the modulation signal need to be lowered. The literature [8] proposes an adaptive PAPR reduction method based on u-law for PSWF modulation signal, which can compress the large amplitude components in the modulation signal, solving the problem that large amplitude component will be limited when they through PA at some level. But signal compression will cause signal distortion, and PA also causes signal distortion, during the dual distortion, the system performance is not high.

To improve BER performance of system and reduce the nonlinear effects of PA, considering the advantage of adaptive PAPR reduction method can effectively lower large amplitude component, and the advantage of predistortion algorithm can effectively compensate the nonlinear distortion of small value component, our team combine PAPR reduction method with predistortion algorithm, at the same time, solving the problem of large amplitude weight will be limit when they through PA. Considering nonlinear distortion is small when small amplitude component through PA, and the compressed signal contains a large number of small amplitude component. At the same, the LUT predistortion algorithm based on compression quantitative [5] can effectively compensate the nonlinear distortion with lower ROM storage demand conditions through reasonable distribution of address.

Based on the above analysis, a new joint RF PA linearization method is proposed, combining adaptive PAPR reduction method based on u-law with the LUT predistortion algorithm based on compression quantitative, its structure diagram is shown in Fig. 1. The proposed method places PAPR reduction method in series with the LUT predistortion algorithm, first compress the signal amplitude by using PAPR reduction method, then linearize the modulation signal by using the LUT predistortion algorithm, solving the weakness of the existing predistortion algorithm that predistortion algorithm become invalid when large amplitude component through PA.

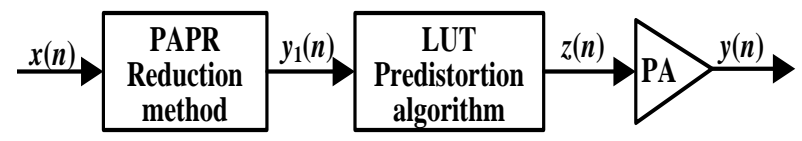

Figure 1. The model of joint PA linearization method.

\section{B. The System of Joint RF Power Amplifier Linearization Method}

In section 2.1, we analysis the model of joint PA linearization method, then we design the joint predistortion method system scheme, its system model is shown in Fig. 2, PA linearization by the method in this paper includes two processes, one is signal compression processing, the other is signal predistortion processing. The main steps are as follows:

Compress the large amplitude components in the signal by using PAPR reduction method. Calculate the parameter A by using calculate $\mathrm{E}(|\mathrm{x}|)$ module in Fig. 2, according to the following formula.

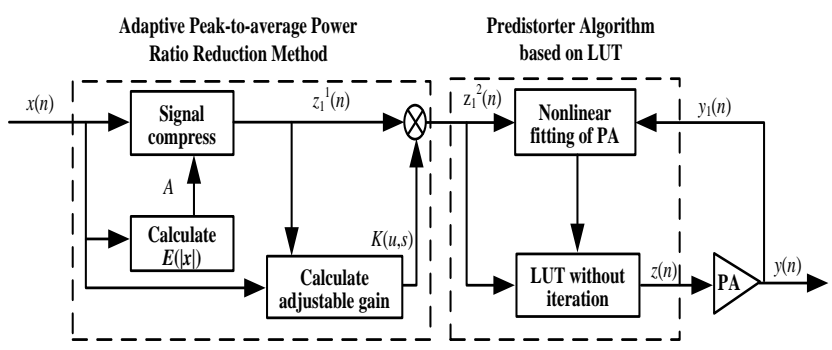

Figure 2. The system of joint PA linearization method.

$$
A=\frac{1}{N} \sum_{i=0}^{N-1}|x(n)|
$$

Where, $\mathrm{x}(\mathrm{n})$ is amplitude of the input signal, $\mathrm{N}$ is the sampling numbers. Adjust the compression parameters automatically through calculating $\mathrm{E}(|\mathrm{x}|)$, according to the input signal.

- (2) Calculate compressed signal z11(n) by using signal compress module in Fig. 2, according to the following formula.

$$
z_{1}^{1}(n)=\frac{A \cdot \ln \left(1+\mu \frac{|x(n)|}{A}\right)}{\ln (1+\mu)} \cdot \operatorname{sgn}(x(n)), n=0,1,2 \ldots, N-1
$$

Where, $\operatorname{sgn}(\cdot)$ is symbolic function, $\mathrm{x}(\mathrm{n})$ is compression parameter of $\mathrm{u}$-law, which needs to be set according to actual situation. For example, we can let $\mathrm{u}=3$, under the simulation conditions in section 3.1. 
- (3)Calculate adjustable gain $K(\mu, s)$ by using calculate adjustable gain module in Fig. 2, according to the following formula.

$$
K^{2}(u, s)=\sum_{i=0}^{N-1} \frac{|x(n)|^{2}}{\frac{\left(\mathrm{A} \ln \left(1+\frac{\mu|x(n)|}{A}\right)\right)^{2}}{(\ln (1+\mu))^{2}}}
$$

Adjust adjustable gain $\mathrm{K}(\mu, \mathrm{s})$ automatically according to the input signal, ensuring signal average power remains the same before and after compression.

- (4) Calculate the output signal z12(n) of PAPR reduction method by using multiplication module in Fig. 2, according to the following formula.

$$
y(n)=K(u, s) \cdot \frac{A \cdot \ln \left(1+\mu \frac{|x(n)|}{A}\right)}{\ln (1+\mu)} \cdot \operatorname{sgn}(x(n)), n=0,1,2 \ldots, N-1
$$

- (5) Complete the construction of the look-up table of predistortion algorithm based on compressing quantization[5]. Complete reasonable distribution of address, according the characteristics of PSWF modulation signal amplitude.

- (6) Calculate the final output signal $z$ (n) of predistortion algorithm by using LUT without iteration module in Fig. 2.

\section{SIMULATION RESULTS}

From PSD of modulation signal and BER performance of the system, comparing with amplitude limiting, LUT predistortion algorithm based on compression quantitative, adaptive PAPR reduction method, to verify the feasibility and performance of joint PA linearization method proposed in this paper.

\section{The Simulation Conditions}

\section{1) Modulation signal:}

frequency band $1 \mathrm{MHz} 1.1 \mathrm{MHz}$, divide into four subsystems wave band, the frequency spectrum overlapping degree is $50 \%$, time bandwidth product $\mathrm{c}=4 \mathrm{~Hz} \cdot \mathrm{s}$. Each wave band take the first two order PSWF pulses according to the energy from high to low, generating non-sinusoidal orthogonal modulation signal by overlaying PSWF pulses, and normalizing signal amplitude normalization, then linear gain 1.2 times.

2) Power amplifier:

Saleh model, its AM-AM and AM-PM features of Saleh model are expressed as in formula (1). Where, $\alpha \mathrm{a}=2, \beta \mathrm{a} 2=1$, $\alpha \psi=\pi / 3, \beta \psi=1[9]$.

\section{The Results and Analysis of Simulation Results}

1) PSD of modulation signal:

The PSD of modulation signal reflects the out-of-band distortion compensation ability of predistortion algorithm, the PSD of PSWF modulation signal is shown in Fig. 3. As can be seen from the bar chart, the out-of-band distortion of PSWF modulation signal about $20 \mathrm{~dB}$ when PSWF modulation signal directly through $\mathrm{PA}$, and predistortion algorithm has been unable to compensate out-of-band distortion of modulation signal. After dealing with the method in this paper, then PSWF modulation signal through PA, effectively improving the power spectrum of the signal with about $5 \mathrm{~dB}$, and the out-of-band distortion compensation ability of the proposed method is close to adaptive PAPR reduction method, better than amplitude limiting and LUT predistortion algorithm based on compression quantitative.

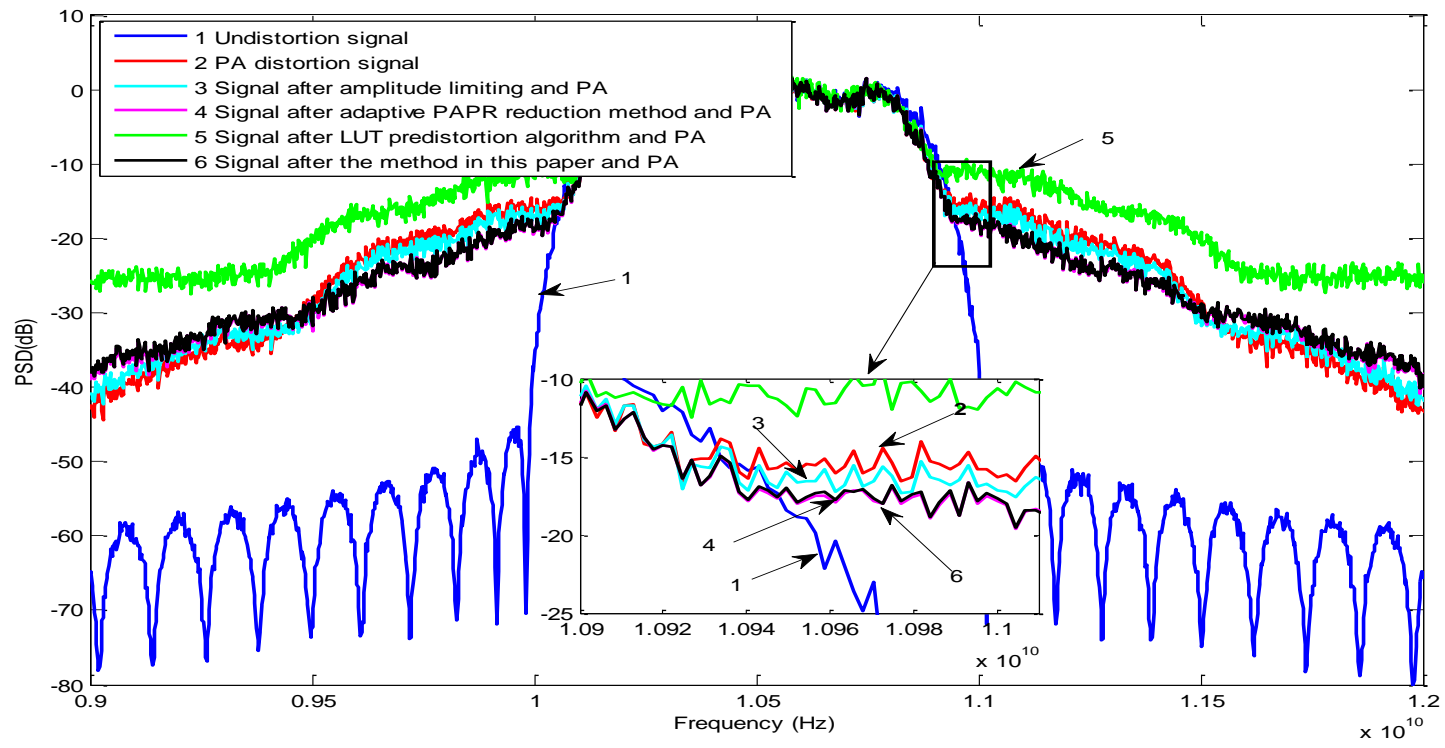

Figure 3. PSD of modulation signal. 


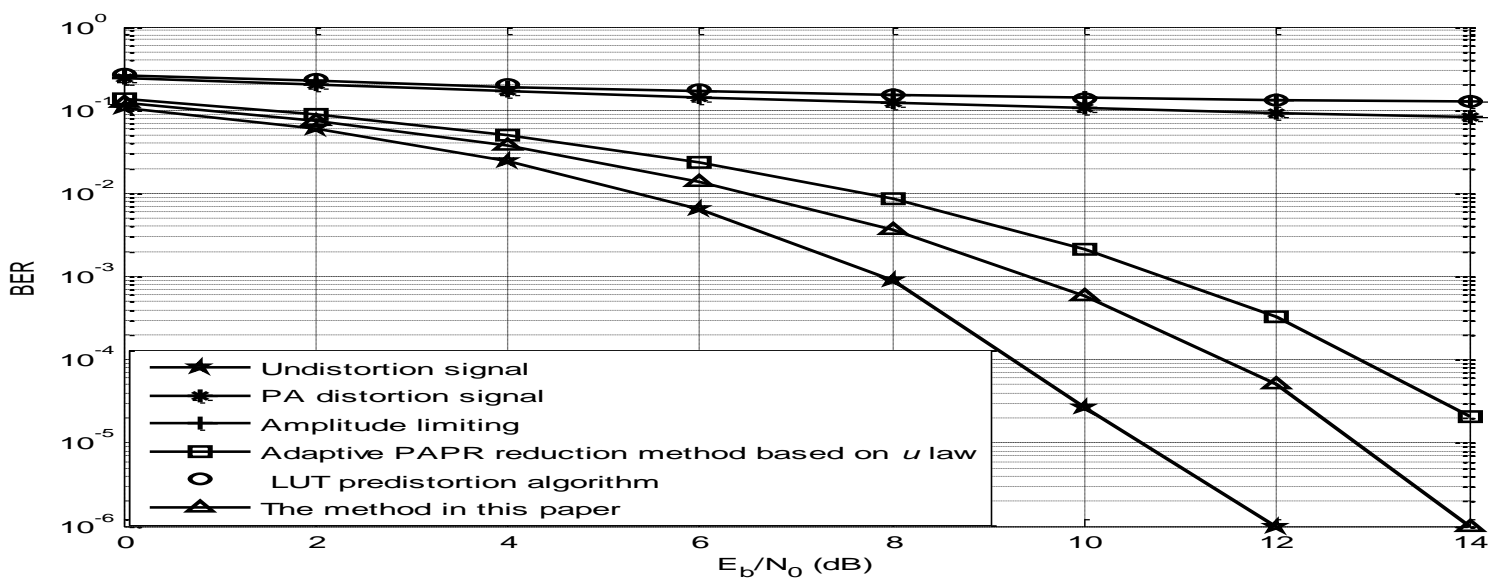

Figure 4. BER performance in nonlinear AWGN channel.

2) BER performance in nonlinear AWGN channel:

BER performance is an important indicators to measure the reliability of communication system, reflects in-band distortion compensation ability of predistortion algorithm. Fig. 4 depicts the performance of BER versus Eb/N0 of PSWF modulation signal by different PA nonlinear suppression method over the AWGN channel. From Fig. 4, it is observed that the method in this paper can effectively improve the BER performance of system, and reduce the gap to the original signal less than $2 \mathrm{~dB}$ when the BER performance is 10-5 in nonlinear AWGN channel. At the same time, the BER performance of system which uses the method in this paper better than the system which uses amplitude limiting, adaptive PAPR reduction method and LUT predistortion algorithm based on compression quantitative.

\section{SUMMARY}

In this paper, a new joint RF PA linearization method is proposed, combining PAPR reduction method base on u-law the LUT predistortion algorithm based on compression quantitative, making full use of advantage of the two boxes, solving the weakness of the existing predistortion algorithm, when large amplitude component in the modulation signal which is more than the voltage in saturation will be limited, leading to predistortion algorithm become invalid. The simulation result shows that the proposed method can effectively improve the power spectrum of and the BER performance of system. How to optimize the parameter Settings of PAPR reduction method and LUT predistortion algorithm which are used in the proposed method in this paper, implementing to ensure system performance unchanged and reduce the algorithm complexity will be the next study focus.

\section{ACKNOWLEDGEMENT}

The authors would like to thank the National Science Foundation. The author would also link to thank the editor and the anonymous reviewers for their constructive and comments during the review process which had helped to improve the quality of the paper.

\section{REFERENCES}

[1] Flammer C. Spheroidal Wave Functions[M]. California: Stanford University Press, 1956: 16-19.

[2] Zhao Z Y, Wang H X, Liu X G, et al. Orthogonal Prolate Spheroidal Wave Functions Modulation Method[J]. Journal of Electronics \& Information Technology, 2012, 34(10): 2331-2335.

[3] Osipove A, Rokhlin V. On the Evaluation of Prolate Spheroidal Wave Functions and Associated Quadrature Rules[J]. Appliedand Computational Harmonic Analysis, 2014, 36: 108-142.

[4] Wang H X, Zhao Z Y, and Liu X G, et al. The Nonsinusoidal Orthogonal Modulation in Time Domain[P]. China, ZL2008159238.3, 2011-02-02.

[5] Zong P L, Wang H X, Sun X D, Pan Y Z. Predistortion Algorithm Based on Compressing Quantization for Nonsinusoidal Orthogonal Modulation Signal in Time-domain[J], Journal of Electronics \& Information Technology, 2013, 35(3): 658-664.

[6] Zhong P L, Wang H X, Liu C H, et al. A Predistortion Algorithm Based on Parallel Two-box Model[J]. Journal of Hua zhong University of Science and Technology (Nature Science Edition), 2014, 42(2): $86-90$

[7] Wang H X, Lu F P, Liu C H, et al. Predistortion Algorithm Based on RLS for PSWF Orthogonal Modulation Signal[J]. Journal of China Academy of Electronics and Information Technology, 2016, 11(1): 98-102.

[8] Wang H X, Lu F P, Liu C H, et al. An Adaptive Peak-to-average Power Ratio Reduction Method for PSWF Orthogonal Modulation Signal[J]. Journal of Electronics \& Information Technology, 2016.

[9] Saleh, A.A.M. Frequency-independent and frequency-dependent nonlinear models of TWT amplifiers[J]. IEEE Trans Communications, 1981: $1715-1720$ 